\title{
Clinical significance of GADD153 expression in stage I non-small cell lung cancer
}

\author{
CHANG YOUL LEE ${ }^{1}$, MYUNG GOO LEE ${ }^{1}, \mathrm{KYUNG} \mathrm{CHAN} \mathrm{CHOI}^{2}, \mathrm{HEE} \mathrm{MO} \mathrm{KANG}^{3}$ and YOON SOO CHANG \\ Departments of ${ }^{1}$ Internal Medicine and ${ }^{2}$ Pathology, Chuncheon Sacred Heart Hospital, \\ Hallym University Medical Center; ${ }^{3}$ Department of Information Statistics, Hallym University; \\ ${ }^{4}$ Department of Internal Medicine, Yonsei University College of Medicine, Seoul 135-720, Republic of Korea
}

Received February 20, 2012; Accepted May 29, 2012

DOI: $10.3892 / \mathrm{ol} .2012 .768$

\begin{abstract}
The transcription factor growth arrest and DNA damage-inducible gene 153 (GADD153), also known as $\mathrm{CHOP}$, is considered to function as a proapoptotic molecule. Overexpression of GADD153 leads to cell cycle arrest and/ or apoptosis. However, its clinical implications in non-small cell lung cancer (NSCLC) remain controversial. Therefore, we investigated the expression of GADD153 in stage I NSCLC using immunohistochemistry. Paraffin-embedded tissue sections from 76 patients, who were diagnosed with primary stage I NSCLC and had undergone a curative lung resection, were stained using an anti-GADD153 antibody. The intensity of GADD153 immunostaining was evaluated within the cell membrane and cytoplasm of invasive cancer components. The correlation between the intratumoral expression of GADD153 and various clinical parameters were explored. GADD153 was detected in 29 (38.2\%) cases. No statistically significant difference in expression was demonstrated between stage IA and stage IB tumors (35.0 vs. $39.3 \%$; $\mathrm{P}=0.735)$. The expression of GADD153 was not affected by histological subtypes or histological grades of differentiation. The intratumoral expression of GADD153 did not influence the overall survival rate (53.29 vs. 52.18 months; $\mathrm{P}=0.743$ ) or disease-free survival rate (46.97 vs. 54.19 months; $\mathrm{P}=0.084$ ) of stage I NSCLC patients. However, patients with GADD153 expression demonstrated an improved disease-specific survival rate $(28.80$ vs. 53.85 months; $\mathrm{P}=0.020)$. No patients with GADD153 expression demonstrated distant metastasis $(\mathrm{P}=0.029)$. These data suggest that GADD153 expression may be a valuable prognostic factor of early-stage NSCLC in patients who have undergone curative lung resection.
\end{abstract}

Correspondence to: Dr Yoon Soo Chang, Department of Internal Medicine, Yonsei University College of Medicine, 8th Floor Annex Building, Gangnam Severance Hospital, 211 Eonju-ro, Gangnam-gu, Seoul 135-720, Republic of Korea

E-mail: yschang@yuhs.ac

Key words: non-small cell lung cancer, growth arrest and DNA damage-inducible gene, immunohistochemistry, prognosis

\section{Introduction}

Non-small cell lung cancer (NSCLC) accounts for approximately $75-80 \%$ of all lung cancers and is the leading cause of cancer-related mortality worldwide $(1,2)$. Twenty percent of stage I and $30 \%$ of stage II NSCLC patients (3) who are treated with curative intent experience recurrence of cancer, which is often incurable at the time of discovery, and have a 5-year survival rate of less than $50 \%$ (1). Despite years of intense efforts to control lung cancer mortality with surgical resection, chemotherapy and radiotherapy, NSCLC remains the leading cause of cancer-related mortality. This clearly indicates an urgent requirement for elucidating the mechanisms of lung cancer carcinogenesis, as well as discovering new approaches for its prevention and treatment.

The transcription factor growth arrest and DNA damage-inducible gene 153 (GADD153), also known as CHOP $(4,5)$, is considered to function as a proapoptotic molecule. GADD153 belongs to the CCAAT/enhancer binding protein $(\mathrm{C} / \mathrm{EBP})$ family of transcription factors. It forms heterodimers with other $\mathrm{C} / \mathrm{EBP}$ family proteins and changes their transcriptional activity $(5,6)$.

GADD153 is ubiquitously expressed at a low level in several cell types, and its expression is induced by a variety of stress factors $(5,7)$, including genotoxic stress, endoplasmic reticulum (ER) stress and nutrient depletion. Stress induces GADD153 expression at transcriptional and post-transcriptional levels $(4,8-11)$. ER stress is induced by environmental conditions that are frequently encountered in cancer growth; therefore, the role of ER stress in carcinogenesis and tumor progression is being actively researched $(12,13)$. Overexpression of GADD153 has been reported to lead to cell cycle arrest and/ or apoptosis $(14,15)$. Disruption of the GADD153 gene has been identified to render cells more resistant to ER stress-induced apoptosis, while exogenous GADD153 is capable of inducing growth arrest and/or apoptosis (14-16). GADD153 is one of the important factors in the death of cancer cells and in the anticarcinogenic process, where it downregulates cell growth and survival rate. Studies have suggested that GADD153 triggers the critical early events leading to the initiation of apoptosis, which are considered to be of significance in the prognosis of early NCSLC (17). In this study, we aimed to determine whether the expression of GADD153 is an indicator of good 
prognosis in stage I NSCLC patients. Consequently, we evaluated GADD153 expression in 76 stage I NSCLC tissue samples and investigated the correlation between expression and clinical and pathological findings.

\section{Materials and methods}

Study population and samples. Tissue specimens were obtained from 76 patients who were diagnosed with stage I primary NSCLC and underwent curative surgical removal of a primary lesion at the Yonsei Medical Center (Seoul, Republic of Korea) between 1995 and 1998. Pathological evaluation was established for histological classification and staging in all patients. No patient underwent radiotherapy or chemotherapy prior to or after surgery until the disease recurred. Of the 76 patients, $56(73.7 \%)$ were male and $20(26.3 \%)$ were female. The age of the patients ranged from 31 to 83 years, with a mean age of $61.8 \pm 9.97$ years, which was similar to the age distribution in our institution's large database of patients with stage I NSCLC (data not shown). All the clinical and pathological information and follow-up data were based on studies from our tumor registry service. The study was reviewed and approved by the institution's Surveillance Committee and their permission was obtained to use tissue blocks and other pertinent information from patient files.

Immunohistochemical staining for GADD153. Paraffin-embedded $4 \mu \mathrm{m}$-thick tissue sections from 76 primary stage I NSCLC samples were stained with mouse monoclonal antibody against human GADD153 (Abcam, Ltd., Cambridge, UK). All sections were deparaffinized in a series of xylene baths and rehydrated using a graded alcohol series. Paraffin sections were retrieved via microwave treatment and treated with $0.3 \%$ hydrogen peroxidase to block endogenous peroxidase activity. The sections were then incubated with the primary anti-GADD153 antibody.

Following incubation, the sections were processed using standard avidin-biotin immunohistochemical techniques according to the manufacturer's instructions (Vector Laboratories, Burlingame, CA, USA). Diaminobenzidine was used as a chromogen and hematoxylin was used for counterstaining. Adjacent normal-appearing bronchial epithelium within each tissue section served as an internal reference. The intensity of GADD153 immunostaining was evaluated within the cell membrane and cytoplasm of invasive cancer components. Cell staining was regarded as positive or negative and all slides were independently evaluated by two pathologists who were unaware of the clinical and pathological information of the subjects. Cancer cells in at least 4 fields were counted at x200 magnification. The GADD153 immunoreactivity level was classified by the proportion of positive cells: $0,<5 \%$ positive cells; $1+, 5-30 \%$ positive cells; $2+, 30-50 \%$ positive cells; $3+$, $>50 \%$ positive cells. The intensity of GADD153 expression was also scored; 0 , negative to weak; 1 , moderate; 2 , strong. The score was the sum of the intensity and the percentage of positive cells. A score of $\leq 1$ was applied as a cut-off point for loss of GADD153 expression.

Statistical analysis. Survival data of all patients were obtained from the Korea National Statistical Office. A two-sample t-test for independent samples and an $\chi^{2}$-test were used for continuous and categorical variables, respectively. The Kaplan-Meier estimator was used to compute survival rate probability as a function of time and the log-rank test was used to compare survival time between the groups. All statistical tests were two-sided. SPSS software (version 15.0) was used throughout and $\mathrm{P}<0.05$ was considered to indicate a statistically significant difference.

\section{Results}

NSCLC patient characteristics. The NSCLC cases included $56(73.7 \%)$ male and $20(26.3 \%)$ female patients (age range, 31-83 years; mean age, 61.8 \pm 9.97 years). The demographic characteristics of the patients are shown in Table I.

GADD153 immunohistochemistry. Immunohistochemical staining of GADD153 in normal and cancer tissue sections was conducted (Fig. 1). Immunoreactivity for the GADD153 antibody was identified primarily in the cytoplasm of cancer cells. However, in the normal lung tissue, bronchial epithelial cells demonstrated weak immunoreactivity. The intensity of GADD153 staining in the cytoplasm was strong, granular and distinct in the lung cancer tissue. The number of cases with GADD153 positive staining was 29 (38.7\%). There was heterogeneity of staining inside the same tumor with sporadic, patchy, focal or diffused patterns. Such intramural heterogeneity made scoring difficult in certain cases, but immunohistochemical staining of GADD153 was stable and reproducible.

Correlation between GADD153 expression and various clinicopathological parameters. A total of 37 (48.7\%) of the 76 stage I NSCLCs were adenocarcinomas, 36 (47.3\%) were squamous cell carcinomas and $3(3.9 \%)$ were other histological types of NSCLC. GADD153 expression was positive in $29(38.2 \%)$ and negative in $47(61.8 \%)$ cases. We were able to classify 64 tissue samples for histological grade of differentiation; $12(54.5 \%)$ out of 22 were well-differentiated, $7(29.2 \%)$ out of 24 were moderately differentiated and $6(33.3 \%)$ out of 18 were poorly differentiated. Analysis of GADD153 expression revealed that expression was not influenced by histological type $(\mathrm{P}=0.674)$ or differential grade $(\mathrm{P}=0.305)$. In a subgroup analysis among subtypes of adenocarcinoma, no differences were observed between bronchioloalveolar carcinomas and other subtypes of adenocarcinoma. The smoking status of the patients did not influence the expression of GADD153, and none of the patients with GADD153 expression experienced distant metastasis of statistical significance $(\mathrm{P}=0.029)$ (Table I).

Survival analysis. The total follow-up period of the patients who were alive at the time of analysis was 5 years. A total of $21(27.6 \%)$ of the 76 patients died during the follow-up period; $11(14.5 \%)$ succumbed to cancer-related events. The 5 -year survival rate probability of the patient population was $72.3 \%$, which is similar to previous results from a large-scale study (18). Overall survival and disease-free survival rate curves did not demonstrate statistically significant differences between NSCLC patients with and without GADD153 expression (Fig. 2). However, the patients with GADD153 
Table I. Stage I NSCLC patient characteristics relative to GADD153 expression.

\begin{tabular}{|c|c|c|c|}
\hline \multirow[b]{2}{*}{ Patient characteristics } & \multicolumn{2}{|c|}{ GADD153 expression in tumor tissues } & \multirow[b]{2}{*}{ P-value } \\
\hline & $\begin{array}{l}\text { Positive } \\
(\mathrm{n}=29)\end{array}$ & $\begin{array}{l}\text { Negative } \\
(\mathrm{n}=47)\end{array}$ & \\
\hline \multicolumn{4}{|l|}{ Gender } \\
\hline Male & 18 & 38 & 0.07 \\
\hline Female & 11 & 9 & \\
\hline \multicolumn{4}{|l|}{ Smoking status } \\
\hline Smoker; packs/year, mean \pm SD & $21 ; 45.0 \pm 28.73$ & $33 ; 35.2 \pm 19.69$ & $0.112 ; 0.311$ \\
\hline Non-smoker & 6 & 4 & \\
\hline Unknown & 2 & 10 & \\
\hline Pathology & & & 0.674 \\
\hline Adenocarcinoma & 16 & 21 & \\
\hline Squamous cell carcinoma & 12 & 24 & \\
\hline Other & 1 & 2 & \\
\hline Histological grade & & & 0.305 \\
\hline Well-differentiated & 12 & 10 & \\
\hline Moderately differentiated & 7 & 17 & \\
\hline Poorly differentiated & 6 & 12 & \\
\hline Unclassified & 4 & 8 & 0.735 \\
\hline \multicolumn{4}{|l|}{ TNM stage } \\
\hline T1N0M0 & 7 & 13 & \\
\hline T2NOM0 & 22 & 34 & \\
\hline Distant metastasis & 0 & 8 & 0.029 \\
\hline \multicolumn{4}{|l|}{$\begin{array}{l}\text { Mean survival time } \\
\text { (months, 95\% CI) }\end{array}$} \\
\hline Disease-free & $54.19(50.736-57.652)$ & $46.97(38.062-55.881)$ & 0.084 \\
\hline Disease-specific & $53.85(50.702-57.002)$ & $28.80(13.895-43.705)$ & 0.020 \\
\hline Overall & $52.18(46.735-57.624)$ & $53.29(49.123-57.447)$ & 0.743 \\
\hline
\end{tabular}

GADD153, growth arrest and DNA damage-inducible gene 153; NSCLC, non-small cell lung cancer; CI, confidence interval.
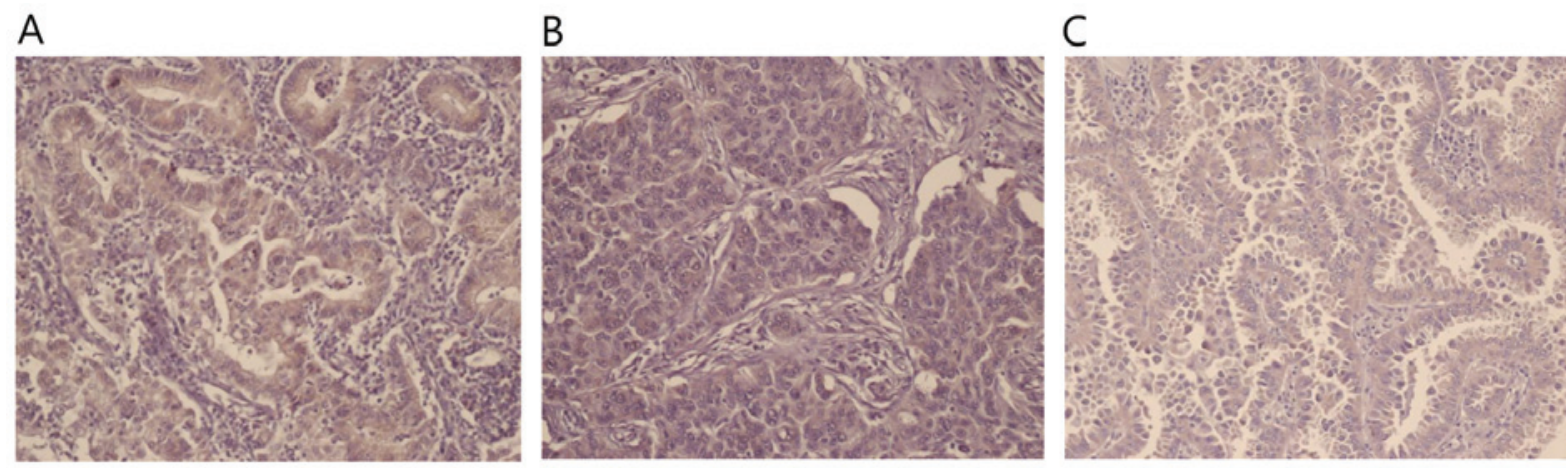

Figure 1. GADD153 expression in NSCLC tissue. (A) Adenocarcinoma and (B) squamous cell carcinoma of the lung demonstrate strong immunoreactivity with GADD153. (C) Bronchioloalveolar carcinoma demonstrates positive GADD153 expression (magnification, x200). GADD153, growth arrest and DNA damage-inducible gene 153; NSCLC, non-small cell lung cancer.

expression had a significantly improved disease-specific survival rate compared to patients with negative GADD153 expression (28.80 vs. 53.85 months; $\mathrm{P}=0.020$ ) (Table I; Fig 2). Subgroup analyses classified according to pathological diagnosis, grade of differentiation or TNM stages demonstrated that GADD153 expression status did not influence disease-free, disease-specific and overall survival rate in NSCLC patients (data not shown). 

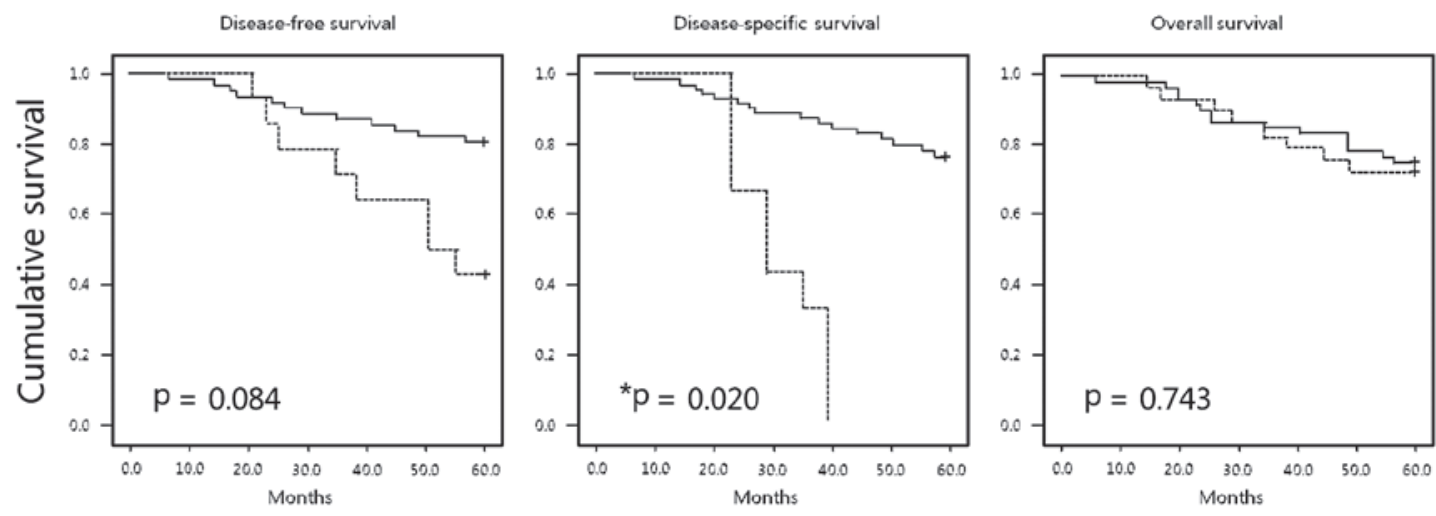

Times to progression (months)

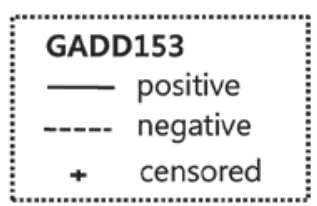

Figure 2. Survival analysis of stage I NSCLC patients according to GADD153 expression. Patients whose tumors expressed GADD153 had a significantly improved disease-specific survival compared to those without GADD153 expression $(\mathrm{P}=0.02)$. GADD153, growth arrest and DNA damage-inducible gene 153; NSCLC, non-small cell lung cancer.

\section{Discussion}

It has been well-established that mutational activation of the Ras gene is a key factor in human cancer development (19). Oncogenic Ras proteins transform cells via multiple downstream signaling cascades, which lead to the phosphorylation and activation of proliferation-inducing transcription factors, including Elk-1, Ets-2 and c-Myc $(19,20)$. Oncogenic Ras has also been identified to downregulate the expression of proapoptotic proteins, including the Bcl-2 family protein Bak and the transcriptional repressor Par-4 (21-24). One study also demonstrated that oncogenic Ras downregulated GADD153 expression at protein and mRNA levels (25).

GADD153, which belongs to the C/EBP family of transcription factors, forms heterodimers with other members of the C/EBP family, resulting in the inhibition of transcriptional activities $(5,17)$. The GADD153 gene is typically induced in response to cellular stress (17). Previously, it has been reported that GADD153 expression may be regulated through various MAP kinase signaling pathways, and that the particular signaling pathway involved is dependent upon the type of stimuli $(16,26,27)$. There is considerable evidence indicating that GADD153 is directly involved in the apoptosis pathway. It has been demonstrated that GADD153 upregulates the proapoptotic factor BH-3 (BIM) and downregulates the antiapoptotic Bcl-2 (28,29). Therefore, GADD153 increases cellular sensitivity to apoptosis by suppressing the transcription of antiapoptotic Bcl-2 (29). Previous studies have demonstrated that oncogenic Ras downregulates GADD153 expression and exogenous GADD153 inhibits Ras-induced cellular transformation (25). Therefore, GADD153 is important not only in killing cancer cells, but also in the anticarcinogenic process, where it downregulates cell growth and survival rate. A previous study has suggested that GADD153 may act as a critical marker of early response to cell injury, and each molecule is known to function in a different signal transduction pathway responsive to cell injury (30). The main role of the GADD153 gene is to block proliferation at $\mathrm{G} 1$ and 2 checkpoints in response to DNA damage. Transfection of the GADD153 gene into various cancer cell lines induces apoptosis without any stress-inducing factors, indicating that GADD153 is directly involved in the regulation of apoptosis (16). Furthermore, the GADD153 protein plays a significant role in the induction of apoptosis of cancer cells treated with $\mathrm{N}$-(4-hydroxyphenyl)retinamide, a synthetic retinoid, in a retinoic acid receptor-independent pathway $(31,32)$. These results strongly suggest that the expression of the GADD153 gene is a new molecular mechanism of antitumor activity. However, studies on the clinical relevance of GADD153 expression in human cancer are extremely limited. In previous studies, increased expression of GADD153 correlated with a lower tumor stage in colon cancer and with a higher survival rate in melanoma $(33,34)$. A further study on lung cancer demonstrated that GADD153 expression correlated with a larger tumor size, higher pathological $\mathrm{T}$ stage, higher TNM stage and shorter overall survival rate (35). Therefore, we focused on the expression of GADD153 in patients with stage I (3) primary NSCLC and its association with clinical outcome. The aim of this study was to examine the expression of GADD153 in stage I NSCLC with respect to prognosis.

We examined the prognostic significance of GADD153 expression in formalin-fixed paraffin-embedded tissues and revealed that GADD153 expression was downregulated in a significant amount of patients with stage I NSCLC. Overall, 29 of 76 tumors (38.7\%) expressed GADD153, and its expression was localized in the membrane and cytoplasm rather than in the nucleus of NSCLC cells. Notably, patients with GADD153 expression demonstrated a positive correlation with improved disease-specific survival rate $(\mathrm{P}=0.020)$. However, our data indicated that GADD153 expression was not associated with an improved overall survival rate and was only slightly associated with greater disease-free survival; no statistically significant differences were identified. We demonstrated that GADD153 expression was closely associated with and may have a role in the prevention of stage I NSCLC distant metas- 
tasis $(\mathrm{P}=0.029)$; thus, the greater disease-specific survival rate in patients with GADD153 expression. Subsequently, we analyzed the association of GADD153 expression with the clinicopathological parameters of stage I NSCLC patients. GADD153 was associated with distant metastasis, but none of the other clinicopathological features of stage I NSCLC patients, suggesting that GADD153 may be involved in apoptotic events preventing metastasis.

Since this study was a preliminary investigation, the number of patients with stage I NSCLC was relatively small (76 patients) and the longest follow-up time was 60 months. This study has revealed that GADD153 expression is a candidate marker that may aid in the stratification of patients according to prognosis following curative surgical removal of a primary lesion. Further comprehensive studies involving the mechanisms that induce expression of GADD153 in NSCLC are required to define the role of GADD153 in lung carcinogenesis. The significant association with survival rate observed in the present study is of particular relevance and should be confirmed in additional cohorts of patients.

\section{Acknowledgements}

This study was supported by the Institutional Grant from Yonsei University College of Medicine (6-2008-0198) provided to YS Chang through the Human Barrier Research Institute.

\section{References}

1. Jemal A, Siegel R, Xu J and Ward E: Cancer statistics, 2010. CA Cancer J Clin 60: 277-300, 2010

2. Greenlee RT, Hill-Harmon MB, Murray T and Thun M: Cancer statistics, 2001. CA Cancer J Clin 51: 15-36, 2001.

3. Sobin L and Wittekind Ch (eds). TNM Classification of Malignant Tumors. 6th edition. Wiley-Liss, New York, pp99-103, 2002:

4. Luethy JD, Fargnoli J, Park JS, Fornace AJ Jr and Holbrook NJ: Isolation and characterization of the hamster gadd153 gene. Activation of promoter activity by agents that damage DNA. J Biol Chem 265: 16521-16526, 1990.

5. Ron D and Habener JF: CHOP, a novel developmentally regulated nuclear protein that dimerizes with transcription factors $\mathrm{C} / \mathrm{EBP}$ and LAP and functions as a dominant-negative inhibitor of gene transcription. Genes Dev 6: 439-453, 1992.

6. Ramji DP and Foka P: CCAAT/enhancer-binding proteins: structure, function and regulation. Biochem J 365: 561-575, 2002.

7. Jousse C, Bruhat A, Carraro V, et al: Inhibition of CHOP translation by a peptide encoded by an open reading frame localized in the chop 5'UTR. Nucleic Acids Res 29: 4341-4351, 2001.

8. Jackman J, Alamo I, Jr and Fornace AJ Jr: Genotoxic stress confers preferential and coordinate messenger RNA stability on the five gadd genes. Cancer Res 54: 5656-5662, 1994.

9. Wang XZ, Lawson B, Brewer JW, et al: Signals from the stressed endoplasmic reticulum induce C/EBP-homologous protein (CHOP/GADD153). Mol Cell Biol 16: 4273-4280, 1996.

10. Bruhat A, Jousse C, Wang XZ, Ron D, Ferrara M and Fafournoux P: Amino acid limitation induces expression of CHOP, a CCAAT/ enhancer binding protein-related gene, at both transcriptional and post-transcriptional levels. J Biol Chem 272: 17588-17593, 1997.

11. Sato N, Urano F, Yoon Leem J, et al: Upregulation of BiP and $\mathrm{CHOP}$ by the unfolded-protein response is independent of presenilin expression. Nat Cell Biol 2: 863-870, 2000.

12. Uramoto H, Sugio K, Oyama T, et al: Expression of endoplasmic reticulum molecular chaperone Grp78 in human lung cancer and its clinical significance. Lung Cancer 49: 55-62, 2005.

13. Hsu WM, Hsieh FJ, Jeng YM, et al: GRP78 expression correlates with histologic differentiation and favorable prognosis in neuroblastic tumors. Int J Cancer 113: 920-927, 2005.

14. Matsumoto M, Minami M, Takeda K, Sakao Y and Akira S: Ectopic expression of CHOP (GADD153) induces apoptosis in M1 myeloblastic leukemia cells. FEBS Lett 395: 143-147, 1996.
15. Barone MV, Crozat A, Tabaee A, Philipson L and Ron D: CHOP (GADD153) and its oncogenic variant, TLS-CHOP, have opposing effects on the induction of G1/S arrest. Genes Dev 8: 453-464, 1994

16. Maytin EV, Ubeda M, Lin JC and Habener JF: Stress-inducible transcription factor $\mathrm{CHOP} / \mathrm{gadd} 153$ induces apoptosis in mammalian cells via p38 kinase-dependent and -independent mechanisms. Exp Cell Res 267: 193-204, 2001.

17. Oyadomari S and Mori M: Roles of CHOP/GADD153 in endoplasmic reticulum stress. Cell Death Differ 11: 381-389, 2004.

18. Martini N, Bains MS, Burt ME, et al: Incidence of local recurrence and second primary tumors in resected stage I lung cancer. J Thorac Cardiovasc Surg 109: 120-129, 1995.

19. Downward J: Targeting RAS signalling pathways in cancer therapy. Nat Rev Cancer 3: 11-22, 2003.

20. Cox AD and Der CJ: The dark side of Ras: regulation of apoptosis. Oncogene 22: 8999-9006, 2003.

21. Rosen K, Rak J, Jin J, Kerbel RS, Newman MJ and Filmus J: Downregulation of the pro-apoptotic protein Bak is required for the ras-induced transformation of intestinal epithelial cells. Curr Biol 8: 1331-1334, 1998.

22. Barradas M, Monjas A, Diaz-Meco MT, Serrano M and Moscat J: The downregulation of the pro-apoptotic protein Par-4 is critical for Ras-induced survival and tumor progression. EMBO J 18: 6362-6369, 1999.

23. Nalca A, Qiu SG, El-Guendy N, Krishnan S and Rangnekar VM: Oncogenic Ras sensitizes cells to apoptosis by Par-4. J Biol Chem 274: 29976-29983, 1999.

24. Qiu SG, Krishnan S, el-Guendy N and Rangnekar VM: Negative regulation of Par-4 by oncogenic Ras is essential for cellular transformation. Oncogene 18: 7115-7123, 1999.

25. Rong R, Montalbano J, Jin W, et al: Oncogenic Ras-mediated downregulation of Gadd153/CHOP is required for Ras-induced cellular transformation. Oncogene 24: 4867-4872, 2005.

26. Wang XZ and Ron D: Stress-induced phosphorylation and activation of the transcription factor CHOP (GADD153) by p38 MAP Kinase. Science 272: 1347-1349, 1996.

27. Scott DW, Mutamba S, Hopkins RG and Loo G: Increased GADD gene expression in human colon epithelial cells exposed to deoxycholate. J Cell Physiol 202: 295-303, 2005.

28. Puthalakath H, O'Reilly LA, Gunn P, et al: ER stress triggers apoptosis by activating BH3-only protein Bim. Cell 129: 1337-1349, 2007.

29. McCullough KD, Martindale JL, Klotz LO, Aw TY and Holbrook NJ: Gadd153 sensitizes cells to endoplasmic reticulum stress by down-regulating $\mathrm{Bcl} 2$ and perturbing the cellular redox state. Mol Cell Biol 21: 1249-1259, 2001.

30. Friedman AD: GADD153/CHOP, a DNA damage-inducible protein, reduced CAAT/enhancer binding protein activities and increased apoptosis in 32D c13 myeloid cells. Cancer Res 56: 3250-3256, 1996.

31. Kim DG, You KR, Liu MJ, Choi YK and Won YS: GADD153mediated anticancer effects of N-(4-hydroxyphenyl)retinamide on human hepatoma cells. J Biol Chem 277: 38930-38938, 2002.

32. Xia Y, Wong NS, Fong WF and Tideman H: Upregulation of GADD153 expression in the apoptotic signaling of $\mathrm{N}$-(4-hydroxyphenyl)retinamide (4HPR). Int J Cancer 102: $7-14,2002$.

33. Korabiowska M, Cordon-Cardo C, Betke H, et al: GADD153 is an independent prognostic factor in melanoma: immunohistochemical and molecular genetic analysis. Histol Histopathol 17: 805-811, 2002.

34. Rask K, Thorn M, Ponten F, et al: Increased expression of the transcription factors CCAAT-enhancer binding protein-beta (C/EBBeta) and C/EBzeta (CHOP) correlate with invasiveness of human colorectal cancer. Int J Cancer 86: 337-343, 2000.

35. Kim KM, Yu TK, Chu HH, et al: Expression of ER stress and autophagy-related molecules in human non-small cell lung cancer and premalignant lesions. Int J Cancer: Sept 27, 2011 (Epub ahead of print). 